\title{
Linking star formation and galaxy kinematics in the massive cluster Abell 2163
}

\author{
Veronica Menacho and Miguel Verdugo
}

Institut fr̈ Astrophysik, University of Vienna, Türkenschanzstr. 17, 1180 Vienna, Austria email: veronica.menacho@univie.ac.at \& miguel.verdugo@univie.ac.at

\begin{abstract}
The origin of the morphology-density relation is still an open question in galaxy evolution. It is most likely driven by the combination of the efficient star formation in the highest peaks of the mass distribution at high- $z$ and the transformation by environmental processes at later times as galaxies fall into more massive halos. To gain additional insights about these processes we study the kinematics, star formation and structural properties of galaxies in Abell 2163 a very massive $\left(\sim 4 \times 10^{15} \mathrm{M}_{\odot}\right.$, Holz \& Perlmutter 2012) merging cluster at $z=0.2$.

We use high resolution spectroscopy with VLT/VIMOS to derive rotation curves and dynamical masses for galaxies that show regular kinematics. Galaxies that show irregular rotation are also analysed to study the origin of their distortion. This information is combined with stellar masses and structural parameters obtained from high quality CFHT imaging. From narrow band photometry $(2.2 \mathrm{~m} / \mathrm{WFI})$, centered on the redshifted $\mathrm{H} \alpha$ line, we obtain star formation rates.

Although our sample is still small, field and cluster galaxies lie in a similar Tully-Fisher relation as local galaxies. Controlling by additional parameters like SFRs or bulge-to-disk ratio do not affect this result. We find however that $\sim 50 \%$ of the cluster galaxies display irregular kinematics in contrast to what is found in the field at similar redshifts $(\sim 30 \%$, Böhm et al. 2004) and in agreement with other studies in clusters (e.g. Bösch et al. 2013, Kutdemir et al. 2010) which points out to additional processes operating in clusters that distort the galaxy kinematics.
\end{abstract}

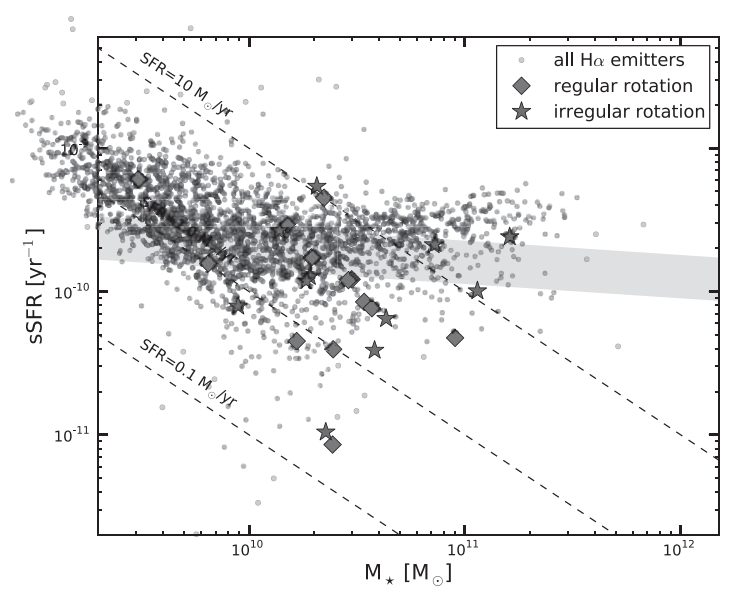

Figure 1. Star forming galaxies in A2163 selected by their $\mathrm{H} \alpha$ emission (small dots). We show in this diagram the cluster galaxies for which we studied their kinematics. Diamonds mark galaxies with regular rotation wheras stars those with irregular kinematics. Dashed lines show constant SFRs whereas the gray area mark the expected sSFR-M $M_{\star}$ relation for this redshift. The most massive galaxy in our sample is a large edge-on spiral clearly expericing ram-pressure stripping as evidenced by its kinematics and trailing star forming regions.

\section{References}

Böhm, A., Ziegler, B. L., Saglia, R. P., et al. 2004, AछA, 420, 97

Bösch, B., Böhm, A., Wolf, C., et al. 2013, A\& A, 554, A97

Holz, D. E. \& Perlmutter, S. 2012, ApJ, 755, L36

Kutdemir, E., Ziegler, B. L., Peletier, R. F., et al. 2010, A\&BA, 520, A109 